\title{
Skewness in the Cosmic Microwave Background Anisotropy from Inflationary Gravity Wave Background
}

\author{
Somnath Bharadwaj \\ Mehta Research Institute,10 Kasturba Gandhi Marg, Allahabad 211 002, India. \\ email: somnath@mri.ernet.in \\ Dipak Munshi \\ Queen Mary and Westfield College, London E1 4NS, United Kingdom \\ email:D.Munshi@qmw.ac.uk \\ Tarun Souradeep \\ Canadian Institute for Theoretical Astrophysics, University of Toronto, Toronto, ON M5S 3H8, Canada \\ email: tarun@cita.utoronto.ca
}

(August 20, 2019)

\begin{abstract}
In the context of inflationary scenarios, the observed large angle anisotropy of the Cosmic Microwave Background (CMB) temperature is believed to probe the primordial metric perturbations from inflation. Although the perturbations from inflation are expected to be gaussian random fields, there remains the possibility that nonlinear processes at later epochs induce "secondary" non-gaussian features in the corresponding CMB anisotropy maps. The non-gaussianity induced by nonlinear gravitational instability of scalar (density) perturbations has been investigated in existing literature. In this paper, we highlight another source of non-gaussianity arising out of higher order scattering of CMB photons off the metric perturbations. We provide a simple and elegant formalism for deriving the CMB temperature fluctuations arising due to the Sachs-Wolfe effect beyond the linear order. In particular, we derive the expression for the second order CMB temperature fluctuations. The multiple scattering effect pointed out in this paper leads to the possibility that tensor metric perturbation, i.e., gravity waves (GW) which do not exhibit gravitational instability can still contribute to the skewness in the CMB anisotropy maps. We find that in a flat $\Omega=1$ universe, the skewness in CMB contributed by gravity waves via multiple scattering effect is comparable to that from the gravitational instability of scalar perturbations for equal contribution of the gravity waves and scalar perturbations to the total $\mathrm{rms}$ CMB anisotropy. The secondary skewness is found to be smaller than the cosmic variance leading to the conclusion that inflationary scenarios do predict that the observed CMB anisotropy should be statistically consistent with a gaussian random distribution.
\end{abstract}

98.80.-k, 98.70Vc, 04.30-w, 04.30Nk, 98.80Bp,

\section{INTRODUCTION}

Since its discovery by Penzias and Wilson [1], the Cosmic Microwave Background (CMB) has proved to be an extremely significant observational guide in our quest towards understanding the universe. The detection of tiny anisotropies in the CMB by the COBE - DMR group [2] was an important milestone in the study of the universe and the understanding the large structure that we see around us. The COBE detection has opened up a fresh avenue of investigation and has been followed by a host of new developments both on the observational and theoretical fronts [3].

The idea of incorporating an inflationary phase in the early universe [4] has gained wide acceptance in the last decade and is perhaps the most prevalent scenario within which one attempts to understand the universe. It was realised soon after the notion of an inflationary scenario was put forward that besides resolving some long standing problems of the Big-Bang model of cosmology, inflationary models also predict the form of the power spectrum of the primordial scalar metric fluctuations which could seed the formation of the large scale structures observed in the present universe [5n]. In fact, both gravity waves (GW), i.e., tensor metric fluctuations [6], as well as adiabatic density perturbations (related to the scalar metric fluctuations) arise as natural consequences of the inflationary scenario, due to the superadiabatic amplification of zero-point quantum fluctuations occurring during inflation. As gravity waves [7] and scalar density perturbations enter the horizon, they induce distortions in the cosmic microwave background (CMB) through the Sachs-Wolfe effect [8]. The relative contribution of the gravity waves and the adiabatic perturbations is linked to the specific model of inflation [9]. The spectral index of the power spectrum of initial perturbations can be inferred directly from the CMB anisotropy measurements. 
The measurements of the power spectrum of CMB temperature fluctuations have till date been found to be consistent with inflationary scenarios of the early universe. In particular, the spectral index inferred from the COBE - 4year data (see for eg. [10]) is consistent with the near scale-invariant spectrum of fluctuations generically predicted by inflationary scenarios. Another generic prediction of inflation is that the metric perturbations generated are gaussian random fields. At the linear order, the CMB anisotropy produced would reflect the gaussian nature of initial perturbations. However, one cannot apriori deny the possibility that non-linear corrections to the growth of perturbations and to the Sachs-Wolfe effect could induce some non-gaussian features in the CMB even for gaussian initial metric perturbations.

Non-zero skewness is a definite signature of non-gaussianity in a distribution. For gaussian initial perturbations the skewness in the CMB appears only beyond the linear approximation. At the leading order the skewness arises from two distinct effects. The first effect is that initially gaussian metric perturbations become non-gaussian when the lowest order nonlinearity becomes important in the course of their evolution due to gravitational instability. The non-linear component of the metric perturbations are non-gaussian and introduce non-gaussian anisotropies in the CMB through a linear Sachs-Wolfe relation at the corresponding order. The second effect is that the gaussian metric perturbations introduce non-gaussian anisotropies in the CMB due to second order (double) scattering of the photon off the linear order metric perturbations. This arises from the second order terms in the Sachs-Wolfe relation. All previous discussions of skewness in the CMB, have been limited to the the estimation of only the first effect, i.e., nonlinearity (and consequent non-gaussianity) due to gravitational instability [11,12,14. The tensor component of metric perturbations (GW) does not exhibit gravitational instability, consequently the possibility of non-gaussianity in the CMB caused by the GW background has been entirely ignored in previous literature.

In this paper, as an illustration of the second effect, we calculate the CMB skewness produced by a gaussian stochastic linear gravity wave background generated during inflation. In the context of $\Omega=1$, flat FRW models, the magnitude of the effect considered here appears to be comparable to the corresponding estimate of CMB skewness arising from the gravitational instability of scalar metric perturbations [12].

In $\S$ II], we outline the basic formalism involved in estimating the skewness in the CMB and present a very general approach for obtaining higher order corrections to the Sachs-Wolfe effect for a general cosmological perturbation. In the following section (\$III) we estimate the skewness in the CMB anisotropy that would arise from a inflationary gravity wave background for a range of values of the spectral index.

\section{FORMALISM}

In this section, we outline the basic approach and present the derivations of results used in our calculation. The first part of the section contains a brief discussion of the perturbative approach used in estimating non-gaussianity in the CMB anisotropy. The second part gives a compact derivation of the CMB anisotropy arising through the Sachs-Wolfe effect upto second order in the primordial metric fluctuations.

\section{A. Non-gaussianity and Nonlinearity in the CMB anisotropy}

It is possible to address non-linear effects in the CMB within a perturbative framework by expanding the temperature fluctuations, $\Delta T / T$, in orders corresponding to the powers of the initial metric perturbation as :

$$
\frac{\Delta T}{T}=\left(\frac{\Delta T}{T}\right)^{(1)}+\left(\frac{\Delta T}{T}\right)^{(2)}+\left(\frac{\Delta T}{T}\right)^{(3)}+\ldots
$$

Given that the initial metric perturbations from inflation are linear and gaussian, any non-gaussian feature in the CMB maps can only arise from the higher order temperature anisotropy such as $(\Delta T / T)^{(2)}$. We shall call this higher order effect - secondary non-gaussianity. (The term "secondary" is used to denote the effects which take place after recombination. The effects prior to recombination are "primary".)

A non-vanishing skewness is a definite signature of non-gaussianity in a distribution. At the linear order, the mean CMB skewness $C_{3}^{(3)}(0)=\left\langle\left(\Delta T^{(1)} / T\right)^{3}\right\rangle=0$ where \langle\rangle denotes averaging with respect to different realizations of stochastic space-time metric perturbations of the FRW cosmological model which produce $\Delta T / T$. Substituting the expansion (1) into the expression $C_{3}(0)=\left\langle(\Delta T / T)^{3}\right\rangle$, it is clear that the leading order (in powers of the initial linear metric perturbations) contribution is at the fourth order,

$$
C_{3}^{(4)}(0)=3\left\langle\left(\frac{\Delta T}{T}\right)^{(1)}\left(\frac{\Delta T}{T}\right)^{(1)}\left(\frac{\Delta T}{T}\right)^{(2)}\right\rangle .
$$


(In this paper, we deal only with $C_{3}^{(4)}(0)$, and hence the superscript denoting the order has been dropped in the rest of the text). It is clear from the above expression that the mean skewness depends not only on the magnitude of $\Delta T^{(2)} / T$ but also on the extent of correlation of this term with the linear order terms, $\Delta T^{(1)} / T$. For example, in the case of scalar perturbations the second order terms which arise due to the non-linear evolution of density perturbations grows linearly with the expansion of the universe and can attain values $\Delta T^{(2)} / T \approx 0.1 \Delta T^{(1)} / T$ at late times [15]. However, in a flat $\Omega=1$ universe, the linear order term contributes only close to the surface of recombination $\left(\eta \approx \eta_{\text {rec }}\right)$ and the second order term attains its largest value only at late times $\left(\eta \approx \eta_{0}\right)$. Consequently in the final result for the mean skewness, the decay of correlation between the linear and the second order term over this large physical separation $\left(\approx \eta_{o}\right)$ along the line of sight attenuates the effect of the growth of the second order term, leading to a very modest value for $C_{3}(0)$ 12]. It was also pointed out in the same paper that the mean skewness which arises due to weakly non-linear density perturbations is expected to be somewhat larger in models where the linear gravitational potential changes at late times leading to a significant linear order integrated Sachs-Wolfe contribution at late times (eg. $\mathrm{CDM}+\Lambda, \Omega \neq 1$ models).

Even in a flat $\Omega=1$ universe, contribution to linear order integrated Sachs-Wolfe effect comes from inflationary tensor perturbations (gravity waves). Consequently, one expects that the correlation between the linear and second order terms is not attenuated in this case leading to larger values of $C_{3}(0)$. The second order $\Delta T^{(2)} / T$ in the case of gravity waves comes only from double scattering since GW do not exhibit any gravitational instability. Scalar perturbations also give rise to second order anisotropy through double scattering. However, for flat, $\Omega=1$ models the contribution to the mean skewness is expected to be even smaller than that from gravitational instability considered in [12]. This can be seen from the fact that $\Delta T^{(2)} / T$ from double scattering too has contributions only at late times implying attenuated correlation with the linear term contribution close to the surface of recombination.

\section{B. Second order CMB anisotropy from the Sachs-Wolfe effect}

In a perfectly isotropic universe the $\mathrm{CMB}$ would have the same temperature in all directions on the celestial sphere. If, however, the cosmological metric is perturbed, the temperature observed today fluctuates over the celestial sphere.

The dominant contribution at large angular scales $\left(\theta>1^{\circ}\right)$ to the observed temperature fluctuations comes from the change in the frequency of any CMB photon as it travels from the surface of last scattering to us 7 . In the case of an isotropic universe, the overall increase in the scale factor $a\left(\eta_{0}\right) / a\left(\eta_{\mathrm{rec}}\right)$ redshifts the entire Planckian distribution of photons leading to a Planckian distribution at a lower temperature given $T_{\text {rec }} / T_{0}=a\left(\eta_{0}\right) / a\left(\eta_{\text {rec }}\right)$. The presence of the perturbations $h_{a b}(\eta, \mathbf{x})$, produces an additional change in the frequency and direction (momentum) of a photon as it moves in and out of the fluctuating metric perturbations.

We consider the trajectory of a photon (or ray) in a perturbed flat FRW universe and work in a synchronous coordinate system where the line element is of the form

$$
d s^{2}=a(\eta)^{2}\left[-d \eta^{2}+\left(\delta_{a b}+h_{a b}(\eta, x)\right) d x^{a} d x^{b}\right] .
$$

In the above, $h_{a b}=h_{a b}^{(1)}(\sim \epsilon)+h_{a b}^{(2)}\left(\sim \epsilon^{2}\right)$ is the metric perturbation, and $\epsilon \ll 1$ is a small number characterizing the amplitude of deviations from the unperturbed background FRW universe.

The photon trajectory can be obtained by perturbatively solving the eikonal equation for the phase $S(x, \eta)$. The eikonal equation for a photon propagating in a spacetime with metric, $g_{\mu \nu}$, is

$$
\frac{\partial S}{\partial x^{\mu}} \frac{\partial S}{\partial x^{\nu}} g^{\mu \nu}=0
$$

(analogous to the Hamilton-Jacobi equation for a massive particle). The frequency and the direction of the photon can be obtained from the phase $S(x, \eta)$ using

$$
\omega(x, \eta)=-\frac{1}{a(\eta)} \frac{\partial}{\partial \eta} S(x, \eta), \quad k_{a}(x, \eta)=\nabla_{a} S(x, \eta) .
$$

Retaining terms to order $\epsilon^{2}$ when inverting the metric given by the line element in equation.(3), the eikonal equation (6) becomes

\footnotetext{
${ }^{1}$ We assume instantaneous recombination which is an excellent approximation to standard recombination for calculating the CMB anisotropy at large angular scales $\left(\theta>1^{\circ}\right)$.
} 


$$
-\left(\frac{\partial}{\partial \eta} S\right)^{2}+\left(\delta^{a b}-h^{(1)^{a b}}-h^{(2)^{a b}}+h^{(1)^{a}}{ }_{c}^{(1)^{c b}}\right) \nabla_{a} S \nabla_{b} S=0 .
$$

where we use the background spatial metric $\delta_{a b}$ to raise and lower the spatial indices. The phase $S(x, \eta)$ can be expressed in a perturbative expansion in powers of $\epsilon$, as

$$
S=S^{(0)}+S^{(1)}(\sim \epsilon)+S^{(2)}\left(\sim \epsilon^{2}\right)
$$

and substituting the above in equation (6) we obtain the zeroth order equation

$$
-\left(\frac{\partial}{\partial \eta} S^{(0)}\right)^{2}+\nabla_{a} S^{(0)} \nabla^{a} S^{(0)}=0
$$

the first order equation

$$
-\frac{\partial}{\partial \eta} S^{(0)} \frac{\partial}{\partial \eta} S^{(1)}+\nabla_{a} S^{(0)} \nabla^{a} S^{(1)}=\frac{1}{2} h^{(1)}{ }^{a b} \nabla_{a} S^{(0)} \nabla_{b} S^{(0)},
$$

and the second order equation

$$
\begin{aligned}
-\frac{\partial}{\partial \eta} S^{(0)} \frac{\partial}{\partial \eta} S^{(2)}+\nabla_{a} S^{(0)} \nabla^{a} S^{(2)} & =\frac{1}{2}\left(\frac{\partial}{\partial \eta} S^{(1)}\right)^{2}-\frac{1}{2} \nabla_{a} S^{(1)} \nabla^{a} S^{(1)}+h^{(1)^{a b}} \nabla_{a} S^{(1)} \nabla_{b} S^{(0)} \\
& +\frac{1}{2}\left[h^{(2)^{a b}}-h^{(1)^{a c}} h^{(1)^{b}} c\right] \nabla_{a} S^{(0)} \nabla_{b} S^{(0)}
\end{aligned}
$$

The solutions to these equations correspond to a family of rays (or null geodesics) in a perturbed FRW universe. The solution to the zeroth order solution is

$$
S^{(0)}(x, \eta)=k_{a} x^{a}-\eta+C .
$$

This corresponds to a family of trajectories for the photons with frequency $1 / a(\eta)$ moving in the $\mathbf{k}$ direction. In figure 1, we show some of these trajectories for the case where $\mathbf{k}$ is along the $\mathbf{x}$ axis (the $\mathbf{y}$ direction has not been shown). The observer $\mathrm{O}$ sits at the origin of the spatial coordinate system and measures the frequency of the photons at the present epoch $\eta_{0}$. Using $\lambda$ as a parameter along the rays, this family of rays can be expressed as

$$
\mathbf{x}(\lambda)=\mathbf{k} \lambda+\mathbf{X}
$$

and

$$
\eta(\lambda)=\lambda+\eta_{\text {in }}
$$

with the condition that $\mathbf{X}$ is perpendicular to $\mathbf{k}$. Here different values of $\left(\eta_{\text {in }}, \mathbf{X}\right)$ correspond to different rays and $\left(\eta_{0}, 0\right)$ corresponds to the ray that the observer $\mathrm{O}$ sees at present traveling in the $\mathbf{k}$ direction .

Substituting the first order solution in equation (9), we rewrite the LHS as a derivative along the zeroth order trajectory, i.e., with respect to the parameter $\lambda$ as

$$
\frac{d}{d \lambda} S^{(1)}(x(\lambda), \eta(\lambda))=\frac{1}{2} h_{a b}^{(1)}(x(\lambda), \eta(\lambda)) k^{a} k^{b}
$$

which can be easily integrated to obtain the first order solution,

$$
S^{(1)}\left(\eta\left(\lambda_{1}\right), x\left(\lambda_{1}\right)\right)=\frac{1}{2} \int_{0}^{\lambda_{1}} h_{a b}^{(1)}(\eta(\lambda), x(\lambda)) k^{a} k^{b} d \lambda .
$$

The frequency of the photon is given by the time derivative of $S^{(1)}$ given by equation $(15)$. At the point A (refer to figure 1) the time derivative is the $\Delta \eta \rightarrow 0$ limit of

$$
\frac{S(B)-S(A)}{\Delta \eta}
$$

where $\Delta \eta$ is the difference in $\eta$ between the points $\mathrm{B}$ and $\mathrm{A}$. 
The point A lies on the trajectory $\mathbf{x}=\mathbf{k} \lambda, \eta=\lambda+\eta_{0}$ and it corresponds to a value $\lambda_{1}$ for the parameter $\lambda$. The point $\mathrm{B}$ lies on a different trajectory $\mathbf{x}=\mathbf{k} \lambda, \eta=\lambda+\eta_{0}+\Delta \eta$ and it corresponds to the same value of the parameter i.e., $\lambda_{1}$. We then have

$$
S(B)-S(A)=\frac{1}{2} \int_{0}^{\lambda_{1}}\left[h_{a b}^{(1)}(\eta(\lambda)+\Delta \eta, x(\lambda))-h_{a b}^{(1)}(\eta(\lambda), x(\lambda))\right] k^{a} k^{b} d \lambda
$$

which leads to

$$
\frac{\partial}{\partial \eta} S^{(1)}\left(\eta\left(\lambda_{1}\right), x\left(\lambda_{1}\right)\right)=\frac{1}{2} \int_{0}^{\lambda_{1}} \frac{\partial}{\partial \eta} h_{a b}^{(1)}(\lambda) k^{a} k^{b} d \lambda
$$

Similarly, in the direction parallel to $\mathbf{k}$ (the $x$-axis in fig.11), the spatial derivative is given by limit

$$
\frac{S(C)-S(A)}{\Delta x}
$$

where $\Delta x$ is the difference in $x$ between the points $\mathrm{C}$ and $\mathrm{A}$. $\mathrm{C}$ lies on a trajectory $\mathbf{x}=\mathbf{k} \lambda, \eta=\lambda+\eta_{0}-\Delta x$ and it corresponds to the value of the parameter $\lambda_{1}+\Delta x$. We obtain

$$
S(C)-S(A)=\frac{1}{2} \int_{0}^{\lambda_{1}+\Delta x} h_{a b}^{(1)}(\eta(\lambda)-\Delta x, x(\lambda)) k^{a} k^{b} d \lambda-\frac{1}{2} \int_{0}^{\lambda_{1}} h_{a b}^{(1)}(\eta(\lambda), x(\lambda)) k^{a} k^{b} d \lambda,
$$

and the spatial derivative in the direction parallel to $\mathbf{k}$ reads

$$
\nabla_{c} S^{(1)}\left(\eta\left(\lambda_{1}\right), x\left(\lambda_{1}\right)\right)=\frac{k_{c}}{2}\left[h_{a b}^{(1)}\left(x\left(\lambda_{1}\right), \eta\left(\lambda_{1}\right)\right) k^{a} k^{b}-\int_{0}^{\lambda_{1}} \frac{\partial}{\partial \eta} h_{a b}^{(1)}(\lambda) k^{a} k^{b} d \lambda\right] .
$$

In the direction perpendicular to $\mathbf{k}$ we have

$$
\nabla_{c} S^{(1)}\left(x\left(\lambda_{1}\right), \eta\left(\lambda_{1}\right)\right)=\frac{1}{2} \int_{0}^{\lambda_{1}} \nabla_{c} h_{a b}^{(1)}(\lambda) k^{a} k^{b} d \lambda .
$$

Substituting the derivatives of $S^{(1)}$ in the second order equation (10) and using $\nabla_{\perp a}=\nabla_{a}-k_{a} k^{b} \nabla_{b}$ to denote the spatial derivative in the direction perpendicular to $\mathbf{k}$ we obtain

$$
\begin{aligned}
S^{(2)}(x, \eta) & =\int_{0}^{\lambda_{1}} d \lambda\left\{\frac{3}{8} h_{a b}^{(1)}(\lambda) k^{a} k^{b} h^{(1)}{ }_{c d}(\lambda) k^{c} k^{d}-\frac{1}{4} h_{a b}^{(1)}(\lambda) k^{a} k^{b} \int_{0}^{\lambda} \frac{\partial}{\partial \eta} h^{(1)}{ }_{i j}\left(\lambda^{\prime}\right) k^{i} k^{j} d \lambda^{\prime}\right. \\
& +\frac{1}{2}\left[h^{(2)^{i j}}(\lambda)-h^{(1)^{i l}}(\lambda) h^{(1)}{ }_{l}^{j}(\lambda)\right] k_{i} k_{j}+\frac{1}{2} h^{(1)^{a b}}(\lambda) k_{a} \int_{0}^{\lambda} \nabla_{\perp b} h^{(1)}{ }_{i j}\left(\lambda^{\prime}\right) k^{i} k^{j} d \lambda^{\prime} \\
& \left.-\frac{1}{2}\left[\left(\frac{1}{2} \int_{0}^{\lambda} \nabla_{\perp a} h^{(1)}{ }_{i j}\left(\lambda^{\prime}\right) k^{i} k^{j} d \lambda^{\prime}\right)\left(\frac{1}{2} \int_{0}^{\lambda} \nabla_{\perp}{ }^{a} h^{(1)}{ }_{l m}\left(\lambda^{\prime}\right) k^{l} k^{m} d \lambda^{\prime}\right)\right]\right\} .
\end{aligned}
$$

The perturbative solution for $S(\mathbf{x}, \eta)$ can now be used to obtain the frequency of a photon at any point along its trajectory. Although we are interested in a photon that leaves the last scattering surface at $\eta_{\text {rec }}$ and reaches the observer at $\eta_{0}$, it is convenient to consider a photon traveling backwards in time from the observer to the last scattering surface. Keeping terms upto order $\epsilon^{2}$ we find that the photon that is observed at the frequency $\omega_{o}=1 / a\left(\eta_{o}\right)$ left the last scattering surface with the frequency,

$$
\omega_{e}=\frac{1}{a\left(\eta_{e}\right)}\left[1-\frac{\partial}{\partial \eta} S^{(1)}\left(x_{e}, \eta_{e}\right)-\frac{\partial}{\partial \eta} S^{(2)}\left(x_{e}, \eta_{e}\right)\right] .
$$

Invert the above relation we find that a photon which left the last scattering surface with the frequency $\omega_{o}=1 / a\left(\eta_{e}\right)$ will have frequency

$$
\omega_{o}=\frac{1}{a\left(\eta_{o}\right)}\left[1+\frac{\partial}{\partial \eta} S^{(1)}\left(x_{e}, \eta_{e}\right)+\frac{\partial}{\partial \eta} S^{(2)}\left(x_{e}, \eta_{e}\right)+\left(\frac{\partial}{\partial \eta} S^{(1)}\left(x_{e}, \eta_{e}\right)\right)^{2}\right]
$$


when it reaches the observer. This relates the observed frequency to the emitted frequency and the metric perturbations. Using equations (24) and (25) we obtain expressions for the fractional change in the frequency of the observed photon relative to the frequency that would be observed if the universe were unperturbed. Since the CMB photons have a Planckian distribution, (frequency independent) fractional changes in frequency translates to fluctuations in the temperature characterizing the distribution. At the linear order we recover the familiar (linear order) Sachs Wolfe effect

$$
\frac{\Delta T^{(1)}}{T}=-\frac{1}{2} \int_{\eta_{\mathrm{rec}}}^{\eta_{0}} \frac{\partial}{\partial \eta} h_{a b}^{(1)}(x(\lambda), \eta(\lambda)) k^{a} k^{b} d \lambda .
$$

At the second order the expression for fractional change in CMB temperature reads

$$
\begin{aligned}
\frac{\Delta T^{(2)}}{T} & =\int_{\eta_{0}}^{\eta_{\text {rec }}} d \lambda\left\{\frac{3}{4} h_{a b}^{(1)}(\lambda) k^{a} k^{b} \frac{\partial}{\partial \eta} h^{(1)}{ }_{c d}(\lambda) k^{c} k^{d}\right. \\
& -\frac{1}{4} \frac{\partial}{\partial \eta} h_{a b}^{(1)}(\lambda) k^{a} k^{b} \int_{\eta_{o}}^{\lambda} \frac{\partial}{\partial \eta} h^{(1)}{ }_{i j}\left(\lambda^{\prime}\right) k^{i} k^{j} d \lambda^{\prime}-\frac{1}{4} h^{(1)}{ }_{a b}(\lambda) k^{a} k^{b} \int_{\eta_{o}}^{\lambda} \frac{\partial^{2}}{\partial \eta^{2}} h^{(1)}{ }_{i j}\left(\lambda^{\prime}\right) k^{i} k^{j} d \lambda^{\prime} \\
+ & \frac{1}{2}\left[\frac{\partial}{\partial \eta} h^{(2)^{i j}}(\lambda)-2 h^{(1)^{i l}}(\lambda) \frac{\partial}{\partial \eta} h^{(1)^{j}}(\lambda)\right] k_{i} k_{j} \\
+ & \frac{1}{2}\left[\frac{\partial}{\partial \eta} h^{(1)^{a b}}(\lambda) k_{a} \int_{\eta_{o}}^{\lambda} \nabla_{\perp b} h^{(1)}{ }_{i j}\left(\lambda^{\prime}\right) k^{i} k^{j} d \lambda^{\prime}+h^{(1)^{a b}}(\lambda) k_{a} \int_{\eta_{o}}^{\lambda} \frac{\partial}{\partial \eta} \nabla_{\perp} h^{(1)}{ }_{i j}\left(\lambda^{\prime}\right) k^{i} k^{j} d \lambda^{\prime}\right] \\
- & {\left[\left(\frac{1}{2} \int_{\eta_{o}}^{\lambda} \frac{\partial}{\partial \eta} \nabla_{\perp a} h^{(1)}{ }_{i j}\left(\lambda^{\prime}\right) k^{i} k^{j} d \lambda^{\prime}\right)\left(\frac{1}{2} \int_{\eta_{o}}^{\lambda} \nabla_{\perp}{ }^{b} h^{(1)}{ }_{l m},\left(\lambda^{\prime}\right) k^{l} k^{m} d \lambda^{\prime}\right)\right] } \\
+ & {\left[\frac{1}{2} \int_{\eta_{r e c}}^{\eta_{0}} \frac{\partial}{\partial \eta} h^{(1)}{ }_{i j}(\lambda) k^{i} k^{j} d \lambda\right]^{2} . }
\end{aligned}
$$

The expression for $\Delta T^{(2)} / T$ has been independently obtained by other methods [17, 16].

The $\Delta T / T$ calculated here is the fractional increment (or decrement) with respect to the CMB temperature that would be observed in a homogeneous and isotropic universe. In practice an observer would measure the fluctuations in the CMB temperature from different directions in the sky and in order to relate our calculation with what is observed we have to remove any monopole component in the angular distribution of the calculated $\Delta T / T$. In addition, the dipole component of $\Delta T / T$ is usually interpreted as arising from the observers peculiar motion and this is removed before relating the observed $\Delta T / T$ to the primordial fluctuations.

In most previous work on second order CMB anisotropy, only the term depending on $h^{(2)}$ arising from nonlinear gravitational instability of scalar density perturbations in the above expression for $\Delta T^{(2)} / T$ has been considered. The nongaussianity from the $h^{(2)}$ term in $\Delta T^{(2)} / T$ for $\Omega=1$ models has been considered in reference [12, 14. The $h^{(2)}$ term in $\Delta T^{(2)} / T$ for $\Omega<1$ models has been calculated in reference 18 to calculate the fourth order corrections to the power spectrum of CMB anisotropies.

\section{SKEWNESS FROM GRAVITY WAVE}

Gravitational waves can be decomposed into two polarization states denoted by + and $\times$, and the metric perturbation corresponding to a gravitational wave with wavelength $\frac{2 \pi}{q}$ traveling in the $z$ direction can be written as

$$
h_{a b}^{\hat{z}}(\eta, \mathbf{x})=e^{i q z} h_{q}(\eta)\left[a_{q}^{+} e_{a b}^{+}+a_{q}^{\times} e_{a b}^{\times}\right] \frac{A_{G}(q)}{q^{\frac{3}{2}}}
$$

where the quantity $A_{G}^{2}(q) / q^{3}$ is the power spectrum of the GW perturbations. The temporal evolution of the modes of the gravity waves in a $\Omega=1$, matter dominated FRW universe is given by

$$
h_{q}(\eta)=\frac{3}{q \eta} j_{1}(q \eta)
$$


where $j_{1}$ denotes the spherical Bessel function of order one. In eqn. (28), the $a_{q}^{\alpha}$ are the complex amplitudes of the two polarization states (henceforth denoted by the superscript $\alpha$ ) and $e_{a b}^{\alpha}$ are the usual basis tensors for the traceless metric perturbations.

A gravity wave in any arbitrary direction $\hat{n}$ can be obtained by rotating the coordinate system (or equivalently the wave traveling in the $z$ direction) so that the wave travels in the required direction. Denoting the rotation by $R(\hat{n})_{a b}$, we can express a gravitational wave traveling in the $\hat{n}$ direction as $h_{a b}^{\hat{n}}=R_{a}^{c}(\hat{n}) R_{b}^{d}(\hat{n}) h_{a b}^{\hat{z}}$.

Thus we can write metric perturbations corresponding to an isotropic stochastic background GW as

$$
\hat{h}_{a b}(\eta, \mathbf{x})=\int \frac{d q d \Omega_{\hat{n}}}{(2 \pi)^{3}} q^{\frac{1}{2}} h_{q}(\eta) e^{i \mathbf{q x}} R_{a c}(\hat{n}) R_{d b}(\hat{n})\left[a_{\mathbf{q}}^{+} e_{c d}^{+}+a_{\mathbf{q}}^{\times} e_{c d}^{\times}\right] A_{G}(q) .
$$

In the above, $a_{\mathbf{q}}^{\alpha}$ are independent random variables which satisfy the relation

$$
<a_{\mathbf{q}}^{\alpha} a_{\mathbf{q}^{\prime}}^{\beta}{ }^{*}>=\delta^{3}\left(\mathbf{q}-\mathbf{q}^{\prime}\right) \delta^{\alpha \beta} .
$$

where the angular bracket denotes an ensemble average over different realizations of the stochastic GW background.

We substitute the expression (30) for the GW metric perturbation in equations (26) and (27) to calculate the temperature fluctuation in the $\mathrm{CMB}$ at the first and second orders, respectively. At the linear order, the $\Delta T / T$ produced by gravity waves has no monopole or dipole component, but at the second order there is a monopole component which has to be removed. To keep the algebra at a manageable level we use the ensemble average of $\Delta T^{(2)} / T$ instead of the angular average of $\Delta T^{(2)} / T$ to estimate the monopole component and we subtract this contribution from the second order temperature fluctuation predicted by equation (27). We next use this to calculate the skewness.

At the leading order the skewness is given by

$$
C_{3}(0) \equiv\left\langle\left(\frac{\Delta T}{T}\right)^{3}\right\rangle=3\left\langle\left(\frac{\Delta T^{(1)}}{T}\right)^{2}\left(\frac{\Delta T^{(2)}}{T}\right)\right\rangle,
$$

and substituting for expressions for $\Delta T^{(1)} / T$ and $\Delta T^{(2)} / T$ we obtain

$$
\begin{aligned}
C_{3}(0)= & \frac{3^{5}}{2^{5} \pi^{10}} \int_{\eta_{o}}^{\eta_{\mathrm{rec}}} d \lambda_{1} \int_{\eta_{o}}^{\eta_{\mathrm{rec}}} d \lambda_{2} \int_{\eta_{o}}^{\eta_{\mathrm{rec}}} d \lambda_{3} \int_{0}^{\infty} \frac{d q_{1}}{q_{1}} \int_{0}^{\infty} \frac{d q_{2}}{q_{2}} A_{G}^{2}\left(q_{1}\right) A_{G}^{2}\left(q_{2}\right) \frac{j_{1}\left(q_{1} \lambda_{3}\right)}{q_{1} \lambda_{3}} \frac{j_{2}\left(q_{1} \lambda_{1}\right)}{\lambda_{1}} \\
& \frac{j_{2}\left(q_{2} \lambda_{3}\right)}{\lambda_{3}} \frac{j_{2}\left(q_{2} \lambda_{2}\right)}{\lambda_{2}}\left[\left(\frac{3 \pi}{8}\right)^{2} \frac{J_{3}\left(q_{1}\left(\lambda_{3}-\lambda_{1}\right)\right)}{\left(q_{1}\left(\lambda_{3}-\lambda_{1}\right)\right)^{2}} \frac{J_{3}\left(q_{1}\left(\lambda_{3}-\lambda_{2}\right)\right)}{\left(q_{1}\left(\lambda_{3}-\lambda_{2}\right)\right)^{2}}-\frac{j_{2}\left(q_{1}\left(\lambda_{3}-\lambda_{1}\right)\right)}{\left(q_{1}\left(\lambda_{3}-\lambda_{1}\right)\right)^{2}} \frac{j_{2}\left(q_{1}\left(\lambda_{3}-\lambda_{2}\right)\right)}{\left(q_{1}\left(\lambda_{3}-\lambda_{2}\right)\right)^{2}}\right] \\
- & \frac{3^{5}}{2^{5} \pi^{10}} \int_{\eta_{o}}^{\eta_{\mathrm{rec}}} d \lambda_{1} \int_{\eta_{o}}^{\eta_{\mathrm{rec}}} d \lambda_{2} \int_{\eta_{o}}^{\eta_{\mathrm{rec}}} d \lambda_{3} \int_{\lambda_{3}}^{\eta_{0}} d \lambda_{4} \int_{0}^{\infty} \frac{d q_{1}}{q_{1}} \int_{0}^{\infty} \frac{d q_{2}}{q_{2}} A_{G}^{2}\left(q_{1}\right) A_{G}^{2}\left(q_{2}\right) \frac{j_{2}\left(q_{1}\left(\lambda_{3}-\lambda_{1}\right)\right)}{\left(q_{1}\left(\lambda_{3}-\lambda_{1}\right)\right)^{2}} \frac{j_{2}\left(q_{2}\left(\lambda_{4}-\lambda_{2}\right)\right)}{\left(q_{2}\left(\lambda_{4}-\lambda_{2}\right)\right)^{2}} \\
& \frac{j_{2}\left(q_{1} \lambda_{1}\right)}{\lambda_{1}} \frac{j_{2}\left(q_{2} \lambda_{2}\right)}{\lambda_{2}}\left[\frac{j_{2}\left(q_{1} \lambda_{3}\right)}{\lambda_{3}} \frac{j_{2}\left(q_{2} \lambda_{4}\right)}{\lambda_{4}}+\frac{j_{1}\left(q_{1} \lambda_{3}\right)}{q_{1} \lambda_{3}} \frac{q_{2}}{\lambda_{4}}\left(4 \frac{j_{2}\left(q_{2} \lambda_{4}\right)}{\lambda_{4}}-j_{1}\left(q_{2} \lambda_{4}\right)\right)\right] \\
+ & \frac{3^{5}}{2^{5} \pi^{10}}\left[\int_{\eta_{o}}^{\eta_{\mathrm{rec}}} d \lambda_{1} \int_{\eta_{o}}^{\eta_{\mathrm{rec}}} d \lambda_{2} \int_{0}^{\infty} \frac{d q}{q} A_{G}^{2}(q) \frac{j_{2}\left(q\left(\lambda_{2}-\lambda_{1}\right)\right)}{\left(q\left(\lambda_{2}-\lambda_{1}\right)\right)^{2}} \frac{j_{2}\left(q \lambda_{1}\right)}{\lambda_{1}} \frac{j_{2}\left(q_{1} \lambda_{2}\right)}{\lambda_{2}}\right]^{2}+6 C_{2}^{2}(0),
\end{aligned}
$$

where $j_{n}$ and $J_{n}$ denote spherical and ordinary Bessel functions of order $n$, respectively. In the case of matter dominated $\Omega_{0}=1$ models with three relativistic species of neutrinos, $\eta_{\mathrm{rec}}=\eta_{0} / 49.6$. The final results are however completley insensitive to the exact value of $\eta_{\mathrm{rec}}$. It is interesting to note that none of the terms in equation (27) which have spatial derivatives in direction perpendicular to $\mathbf{k}$ contribute to the skewness.

We numerically evaluated the above expression to compute the skewness for different spectral indices of GW power spectrum. A dimensionless skewness parameter $S_{3}$ can be constructed by dividing the skewness, $C_{3}(0)$ by the square of the variance, $C_{2}(0)$. The variance of $\Delta T / T$ that arises due to relic gravity waves from inflation is given by

$$
C_{2}(0)=\frac{3^{2}}{2^{3} \pi^{5}} \int_{\eta_{\circ}}^{\eta_{\mathrm{rec}}} d \lambda_{1} \int_{\eta_{o}}^{\eta_{\mathrm{rec}}} d \lambda_{2} \int_{0}^{\infty} \frac{d q}{q} A_{G}^{2}(q) \frac{j_{2}\left(q\left(\lambda_{2}-\lambda_{1}\right)\right)}{\left(q\left(\lambda_{2}-\lambda_{1}\right)\right)^{2}} \frac{j_{2}\left(q \lambda_{1}\right)}{\lambda_{1}} \frac{j_{2}\left(q_{1} \lambda_{2}\right)}{\lambda_{2}} .
$$

The power spectrum of the initial gravitational wave perturbation is assumed to be a scale-free power law, $A_{G}^{2}(k)=$ $A k^{n_{T}}$, where $n_{T}=0$ corresponds to a scale-invariant spectrum. Power law models of inflation produce gravity waves with $n_{T}<0$. We compute the CMB skewness for a broad range in $n_{T}\left(-0.5 \leq n_{T} \leq 0\right)$. We find that the value of 
$S_{3}$ varies from -1.6 to -3.1 for $n_{T}$ varying from the scale invariant spectrum to $n_{T}=-0.5$. It is interesting to note that the skewness arising from gravitational instability of scalar perturbations is comparable for the same range of tilt ( $S_{3} \approx-2.2$ for scale invariant spectrum [12]).

The corresponding observable quantity for the skewness, $C_{3}(0)$ is the all sky-average $\bar{C}_{3}(0)=$ $(4 \pi)^{-1} \int(\Delta T(\theta, \varphi) / T)^{3} d \Omega$ of one particular realization of the random fluctuations. The value obtained by taking an angular average over one sky would generally differ from the ensemble average over all realizations by the (cosmic) variance of the observed skewness, $\bar{C}_{3}(0)$. The skewness originating due to any effect would have an observable significance if the predicted signal stands above the cosmic variance. This is a fundamental limitation and a minimal requirement. In practice, a detectable signal has to stand above additional variances such as instrumental noise, finite beam width of antennas, incomplete sky coverage etc.

The Cosmic variance can be expressed in terms of an angular integral over the cube of the two-point correlation function, $C_{2}(\theta)$ [19]. Assuming a gaussian approximation for the two-point correlation function, we express

$$
C_{2}(\theta)=C_{2}(0) \exp \left[-\frac{l_{c}\left(l_{c}+1\right) \theta^{2}}{2}\right], \quad C_{2}(0) \approx 3 \times 10^{-5}
$$

where the cut-off, $l_{c}$, in the $\Delta T / T$ angular spectrum at large values of the spherical harmonic eigenvalue, $l\left(l_{c} \approx\right.$ $\eta_{0} / \eta_{\mathrm{rec}} \sim 49$ for $\left.\mathrm{GW}\right)$. Using equation (35), the Cosmic variance, $\delta S_{3}$, for the case of a CMB anisotropy arising from gravity waves is given by $\delta S_{3} \approx 1 /\left(C_{2}(0) l_{c}\right) \approx 670$. The Cosmic variance for the scalar case is around 5 times smaller [12]. The Cosmic variance is larger as the power spectrum tilted away (reddened) from scale invariance for both scalar perturbations and gravity waves. It is clear that in principle the secondary skewness in the CMB for a CDM model $\left(\Omega=1, \Omega_{b}=0.05\right.$ and $H_{0}=50 \mathrm{kms} / \mathrm{s} / \mathrm{Mpc}$. $)$ is unobservable since it is below the cosmic variance.

\section{CONCLUSIONS}

In this work we study a possible source of secondary non-gaussianity in the CMB and reexamine whether initial gaussian metric perturbations (as expected from inflation) should lead to a gaussian CMB anisotropy. We point out that besides nonlinear gravitational instability, secondary non-gaussianity can be induced in the CMB maps due to multiple scattering of CMB photon off metric perturbations. We develop a general method for calculating CMB temperature fluctuations beyond the linear order and calculate the expression for CMB temperature fluctuations at the second order. As an example of skewness from multiple scattering, we estimate the skewness in the CMB that is expected to arise due to a relic inflationary gravity wave background. We show that the magnitude of the effect studied here is comparable to the ones considered in existing literature. However, the signal is much smaller than its cosmic variance and we conclude that gaussian initial perturbation from inflation leads to a gaussian statistics for the observed temperature fluctuations.

\section{ACKNOWLEDGMENTS}

S.B. wishes to thank Rajaram Nityananda for very helpful comments and discussions. D.M. and T.S. are grateful to Varun Sahni and Alexei Starobinsky for encouragement and useful comments. The authors also acknowledge assistance from R. Balasubramanian in preparing the figure.

[1] A. A. Penzias and R. W. Wilson, Astrophys. J.142, 419, (1965).

[2] G. F. Smoot et al., Astrophys. J. Lett. 396, L1, (1992).

[3] J. R. Bond, in Cosmology and Large Scale Structure, Proc. Les Houches School, Session LX, ed. R. Schaeffer, (Elsevier Science Publishers, Netherlands, 1996).

[4] A. A. Starobinsky, A.A., Phys. Lett. B 91, 99, (1990); A. H. Guth, Phys. Rev. D 23, 347, (1981); A. D. Linde, Phys. Lett. B 108, 389, (1982); A. Albrecht and P. J. Steinhardt, Phys. Rev. Lett 48, 1220, (1982).

[5] A. A. Starobinsky, Phys. Lett. 117B, 175, (1982); A. H. Guth and S. Y. Pi, Phys. Rev. Lett. 49, 1110, (1982); S. W. Hawking, Phys. Lett. 115B, 295, (1982); J. M. Bardeen, P. J. Steinhardt and M. S. Turner, Phys. Rev. D 28, 679, (1983). 
[6] L. P. Grishchuk, Zh. Eksp. Teor. Fiz. 67, 825, (1974) [ Sov. Phys. JETP 40, 409 (1975) ]; A. A. Starobinsky, JETP Lett. 30, 719, (1979); L. F. Abbott and M. B. Wise, Nucl Phys. 135B, 279, (1984); B. Allen, Phys. Rev. D 37, 2078, (1988); V. Sahni, Phys. Rev. D 42, 453, (1990).

[7] V. A. Rubakov, M. V. Sazhin and A. V. Veryaskin, Phys. Lett. 115B, 189, (1982); A. V. Veryaskin, V. A. Rubakov and M. V. Sazhin, Astron. Zh. 60, 23 (1983); R. Fabbri and M. D. Pollock, Phys. Lett. 125B, 445, (1983).

[8] R. K. Sachs and A. M. Wolfe, Astrophys. J. 147, 73, (1967).

[9] A. A. Starobinsky, Pis'ma Astron. Zh. 11, 323, (1985) [ Sov. Astron. Lett. 11, 133 (1985)]; R. Davis et al., Phys. Rev. Lett. 69, 1856 (1992); A. R. Liddle and D. H. Lyth, Phys. Lett. B291, 391 (1992); F. Lucchin, S. Mataresse and S. Mollerach, Astrophys. J. 401, L49, (1992); D. Salopek, Phys. Rev. Lett.,69, 3602, (1992); T. Souradeep and V. Sahni, Mod. Phys. Lett. A7, 3541, (1992).

[10] C. Bennet et al., Astrophys. J. 464, L1, (1996); and 4-year DMR references therein.

[11] X. Luo and D. N. Schramm, Phys. Rev. Lett.,71, 1124 (1993).

[12] D. Munshi, T. Souradeep and A. A. Starobinsky, ApJ, 454, 552 (1995).

[13] T. Souradeep, Ph.D. thesis IUCAA, (1995).

[14] S. Mollerach et al., Astrophys. J. 453, 1, (1995).

[15] E. Martinez-Gonzalez, J. Sanz and J. Silk, Phys. Rev. D46, 4193,(1992).

[16] W. Hu, D. Scott and J. Silk, Phys. Rev. D49, 648,(1994).

[17] T. Pyne and S. M. Carroll, Phys. Rev. D 53, 2920, (1995).

[18] J. L. Sanz et al., Astrophys. J., 467, 485, (1996).

[19] M. Srednicki, Astrophys. J., 416, L1, (1993). 


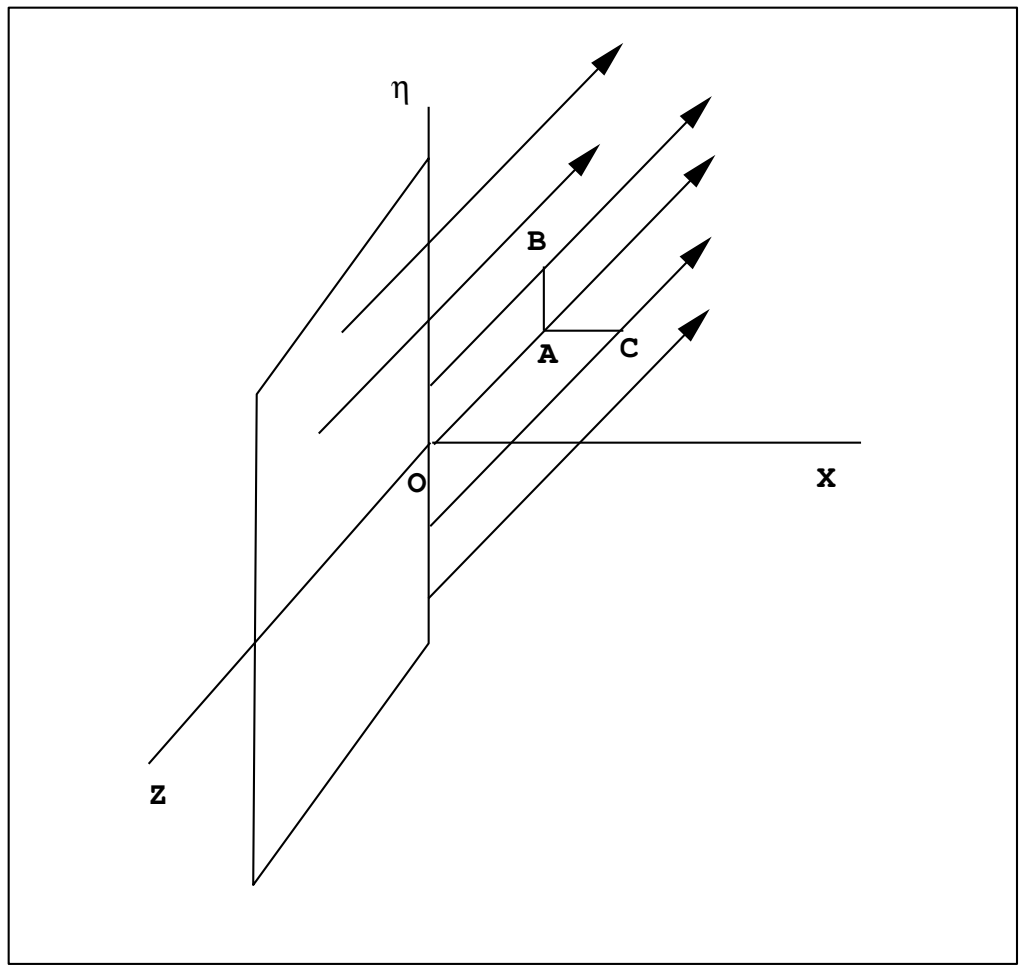

FIG. 1. A sketch of the unperturbed rays of CMB photons propagating along the $\mathbf{x}$ axis is shown (the third spatial dimension is suppressed). The figure serves as a guide to the arguments used in \$I B to compute the temporal and spatial derivatives of the phase $S(\eta, \mathbf{x})$. The figure clearly illustrates the distinction between spatial derivative taken along the direction of photon propagation and spatial derivative taken perpendicular to the direction of photon propagation. 